\title{
Genetic and functional characterization of human pemphigus vulgaris monoclonal autoantibodies isolated by phage display
}

Aimee S. Payne, ${ }^{1}$ Ken Ishii, ${ }^{1}$ Stephen Kacir, ${ }^{2}$ Chenyan Lin, ${ }^{1}$ Hong Li, ${ }^{1}$ Yasushi Hanakawa, ${ }^{1}$ Kazuyuki Tsunoda, ${ }^{3}$ Masayuki Amagai, ${ }^{3}$ John R. Stanley, ${ }^{1}$ and Don L. Siegel${ }^{2}$

${ }^{1}$ Department of Dermatology and 2Department of Pathology and Laboratory Medicine, University of Pennsylvania, Philadelphia, Pennsylvania, USA. ${ }^{3}$ Department of Dermatology, Keio University School of Medicine, Tokyo, Japan.

\begin{abstract}
Pemphigus is a life-threatening blistering disorder of the skin and mucous membranes caused by pathogenic autoantibodies to desmosomal adhesion proteins desmoglein 3 (Dsg3) and Dsg1. Mechanisms of antibody pathogenicity are difficult to characterize using polyclonal patient sera. Using antibody phage display, we have isolated repertoires of human anti-Dsg mAbs as single-chain variable-region fragments (scFvs) from a patient with active mucocutaneous pemphigus vulgaris. ScFv mAbs demonstrated binding to Dsg3 or Dsg1 alone, or both Dsg3 and Dsg1. Inhibition ELISA showed that the epitopes defined by these scFvs are blocked by autoantibodies from multiple pemphigus patients. Injection of scFvs into neonatal mice identified 2 pathogenic scFvs that caused blisters histologically similar to those observed in pemphigus patients. Similarly, these 2 scFvs, but not others, induced cell sheet dissociation of cultured human keratinocytes, indicating that both pathogenic and nonpathogenic antibodies were isolated. Genetic analysis of these mAbs showed restricted patterns of heavy and light chain gene usage, which were distinct for scFvs with different desmoglein-binding specificities. Detailed characterization of these pemphigus $m A b s$ should lead to a better understanding of the immunopathogenesis of disease and to more specifically targeted therapeutic approaches.
\end{abstract}

\section{Introduction}

Pemphigus vulgaris (PV) and pemphigus foliaceus $(\mathrm{PF})$ are potentially fatal autoimmune blistering skin diseases in which autoantibodies against desmoglein 3 (Dsg3) and Dsg1, cell surface desmosomal adhesion molecules, cause loss of keratinocyte cell adhesion (reviewed in ref. 1). PF is characterized by superficial blistering of only the skin, while PV typically presents with suprabasilar blistering of mucous membranes, which may extend to involve skin. ELISA studies have shown that all PF sera contain autoantibodies against Dsg1, and sera from patients with mucosal-dominant PV react mainly against Dsg3 (2-4). PV patients who progress from mucosal to mucocutaneous lesions develop anti-Dsg1 in addition to anti-Dsg3 antibodies (5). The anti-Dsg antibodies in pemphigus sera are pathogenic, since neonatal mouse passive transfer studies have shown that the extracellular domains of Dsg1 and Dsg3 can adsorb out pathogenic antibodies from PF and PV sera, respectively, and affinity-purified anti-Dsg1 or anti-Dsg3 antibodies cause characteristic disease (6-8).

The autoantibody profile in pemphigus patients' sera, together with studies demonstrating the compensatory intercellular adhesive functions of Dsg1 and Dsg3 in normal epidermis (9), accounts for

Nonstandard abbreviations used: $\mathrm{BP}$, bullous pemphigoid; $\mathrm{CDR}$, complementaritydetermining region; D1, scFvs selected against only Dsg1; D3, scFvs selected against only Dsg3; D31, scFvs selected against both Dsg3 and Dsg1; Dsg, desmoglein; ETA, exfoliative toxin A; FR, framework region; IIF, indirect immunofluorescence; $\mathrm{mDsg} 1$, murine Dsg1; mDsg3, murine Dsg3; PF, pemphigus foliaceus; PV, pemphigus vulgaris; PVLIB, PV patient sera used to make the phage display library; PV(3) sera, PV sera containing anti-Dsg3 antibodies; $\mathrm{PV}(3+1)$ sera, $\mathrm{PV}$ sera containing both anti-Dsg3 and anti-Dsg1 antibodies; R/S ratio, ratio of replacement to silent mutations; $\mathrm{scFv}$, singlechain variable-region fragment; $S F M$, serum-free media; $V_{\mathrm{H}}$, variable-region heavy chain; $\mathrm{V}_{\mathrm{L}}$, variable-region light chain.

Conflict of interest: The authors have declared that no conflict of interest exists.

Citation for this article: $J$. Clin. Invest. 115:888-899 (2005).

doi:10.1172/JCI200524185. the clinical and histologic sites of blister formation in pemphigus. In mucous membranes, Dsg1 is expressed predominantly in the superficial epithelium, while Dsg3 is expressed throughout $(10,11)$. In skin, Dsg1 is expressed throughout the epidermis (predominantly superficially), while Dsg3 is expressed only in the basal and immediate suprabasal layers. Thus, consistent with the concept of "desmoglein compensation" (9), in PF, anti-Dsg1 antibodies cause blistering in the superficial epidermis, where Dsg1 but not Dsg3 is expressed, but they do not affect oral mucosa because of compensatory adhesion provided by Dsg3 throughout the epithelium. In mucosal PV, anti-Dsg3 antibodies cause blistering only in the basal layers of the mucosa, where Dsg3 is present without Dsg1 to compensate. The development of anti-Dsg1 in addition to antiDsg3 antibodies in mucocutaneous PV results in the extension of suprabasilar blistering to the epidermis.

Currently, therapy for PV is nonspecific and relies on general suppression of the immune system to ultimately lower antibody titers. To develop more targeted therapies for this disease, a finer understanding of both the $\mathrm{T}$ cell and the $\mathrm{B}$ cell immune response will be needed. A number of studies have examined the role of $\mathrm{T}$ lymphocytes in disease, through MHC-linked susceptibility, TCR gene usage patterns, the identification of $\mathrm{T}$ cell subsets contributing to disease, and the characterization of $\mathrm{T}$ regulatory cells in patients and MHC-matched controls (12-16).

Many more studies have focused on characterizing pemphigus autoantibodies. The relationship of the valence of autoantibodies to pathogenicity has been examined $(17,18)$. Fab' monovalent fragments, prepared by proteolytic degradation and alkylation/ reduction of whole IgG from both PF and PV sera, cause histologically typical disease when passively transferred to neonatal mice. Furthermore, these monovalent antibody fragments may be more potent on a molar basis than bivalent IgG autoantibodies. 
A

(D3) $3 \mathrm{c} / 9$

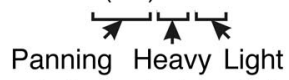
substrate chain chain

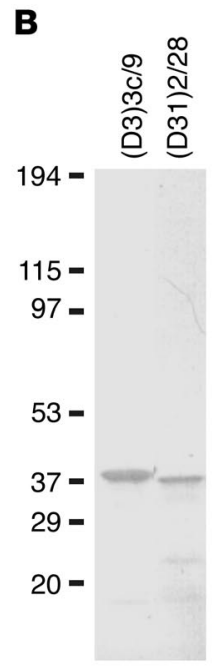

C

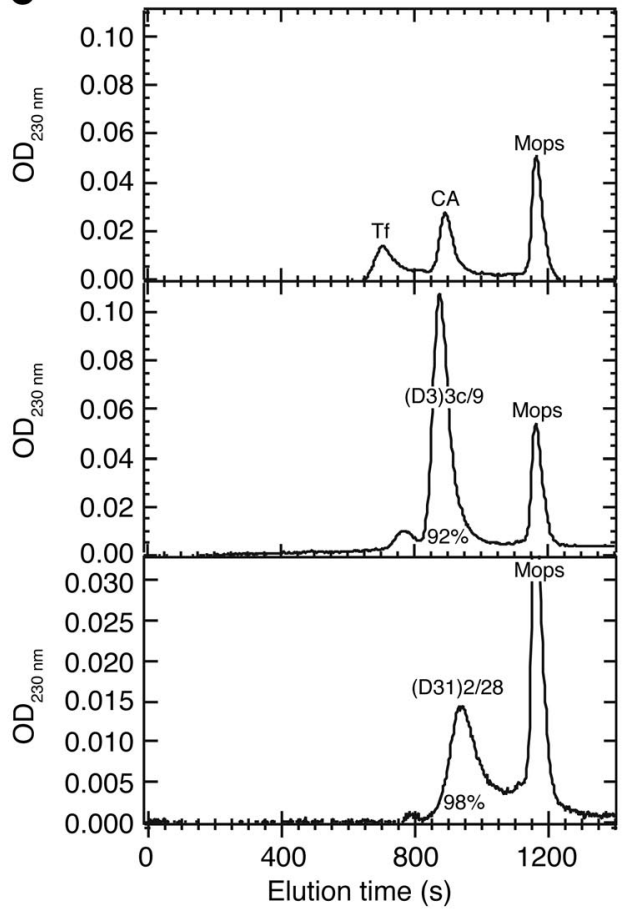

Figure 1

Isolation of monovalent scFv mAbs. (A) ScFv nomenclature. (D3), (D1), or (D31) indicates the antigens used to select anti-Dsg mAbs from the scFv phage display library (Dsg3, Dsg1, or both, respectively). This is followed by a unique heavy chain and light chain nucleotide sequence designation. For mAbs that share the same clonal origin (same VDJ rearrangement) but differ in sequence because of somatic hypermutation, lower-case letters indicate unique members of a given clone (see Discussion and legend to Figure 9). (B) Soluble scFv mAbs were purified by nickel-chelation chromatography (see Methods). Two representative scFvs are shown by Coomassie blue staining after SDS-PAGE. (C) Gel-filtration HPLC demonstrates that scFvs are primarily monomeric in solution. Transferrin (Tf, $75 \mathrm{kDa})$ and carbonic anhydrase (CA, $30 \mathrm{kDa}$ ) served as protein standards, with $0.1 \mathrm{M}$ Mops as a marker of the total volume of the column. The percentage of monomeric protein is shown, calculated from the area under the curve.
These studies demonstrate that neither cross-linking of desmoglein on the keratinocyte cell surface nor fixation of complement is necessary for pathogenicity.

More recent studies have characterized the epitopes on desmogleins that are bound by polyclonal autoantibodies from patients $(19,20)$. These studies indicate that, although antibodies bind to all regions of the extracellular domain of Dsg1 and Dsg3, the predominant epitopes are found at the amino terminus of these molecules. In longitudinal studies of patients with an endemic form of PF, the development of antibodies against the amino terminus of Dsg1 is associated with the onset of active disease. Consistent with those findings, immunoadsorption of PF sera with only the amino-terminal epitopes of Dsg1 is capable of eliminating the ability of the antibodies to cause blisters in neonatal mice.

Taken together, the above data are consistent with the hypothesis that pemphigus antibodies against the amino-terminal adhesive interface of desmogleins may directly interfere with their cell adhesion function. However, with human polyclonal autoantibodies it is difficult to test that hypothesis directly. Recently, mAbs were raised against Dsg3 in PV model mice that were generated by adoptive transfer of lymphocytes from Dsg3-deficient mice to Dsg3-expressing mice $(21,22)$. As Dsg3-deficient mice do not acquire tolerance against Dsg3, lymphocytes from Dsg3-deficient mice efficiently produce IgG against Dsg3 as a nonself protein. These studies showed that a single mAb could induce the PV phenotype, and that this particular antibody bound the predicted amino-terminal adhesive interface of Dsg3 (21). However, PV model mice may not reflect the pathophysiologic mechanisms of disease onset in humans, where production of autoantibodies requires a break in tolerance.

Based on the data and observations discussed above, we concluded that a crucial next step to better understand the pathophysiology of pemphigus autoantibodies and to allow development of more specifically targeted therapies would be more detailed characterization of autoantibodies from patients. Such analysis would be greatly facilitated by isolation of human monoclonal anti-Dsg antibodies from pemphigus patients, which could then be genetically and functionally characterized. To test the feasibility of this approach, we used a powerful molecular technology known as antibody phage display to isolate clones of human anti-Dsg mAbs from a patient with active acute mucocutaneous PV, whose sera contained antibodies to both Dsg3 and Dsg1. This approach obviated the need for the immortalization of human B cells, a process that is efficient for the production of murine mAbs, but not for those of human origin $(23,24)$. Phage display provided us with a large cohort of antiDsg mAbs, which we characterized on a phenotypic level to verify that these recombinant pemphigus $m A b s$ recapitulate both the in vitro and the in vivo desmoglein-binding properties of pemphigus patient sera. We compared their fine specificities with those of antiDsg antibodies present in sera from multiple PV and PF patients to evaluate whether these single-chain variable-region fragment $(\mathrm{scFv})$ $\mathrm{mAbs}$ are representative of autoantibodies from other pemphigus patients. Additionally, we tested our recombinant human anti-Dsg $\mathrm{mAbs}$ in a human keratinocyte cell culture system and a neonatal mouse model for their ability to induce loss of cell adhesion, the hallmark of pemphigus. Together these data suggest that the recombinant pemphigus $m A b s$ reproduce the desmoglein-binding specificities and pathogenicity of polyclonal IgG from multiple pemphigus patients. Analysis of pemphigus $\mathrm{mAb}$ variable-region heavy and light chain gene usage provided evidence for genetic restriction, with antibodies of different desmoglein-binding specificities arising from clonally distinct populations of B lymphocytes. These studies describe a novel method for investigating the generation of autoimmunity and the pathogenesis of disease in PV.

\section{Results}

Isolation of human anti-Dsg3 and anti-Dsg1 mAbs from a phage display library constructed from lymphocytes from a mucocutaneous $P V$ patient. RT-PCR was used to amplify mRNA for the Ig variable-region 

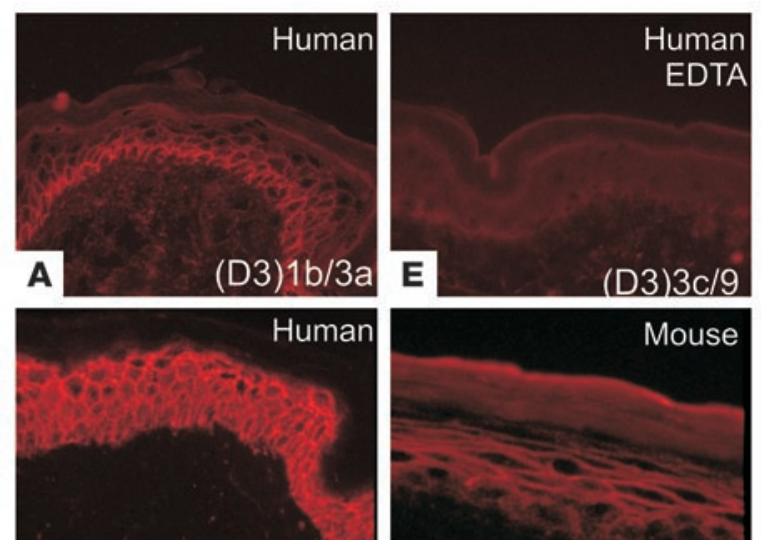

B
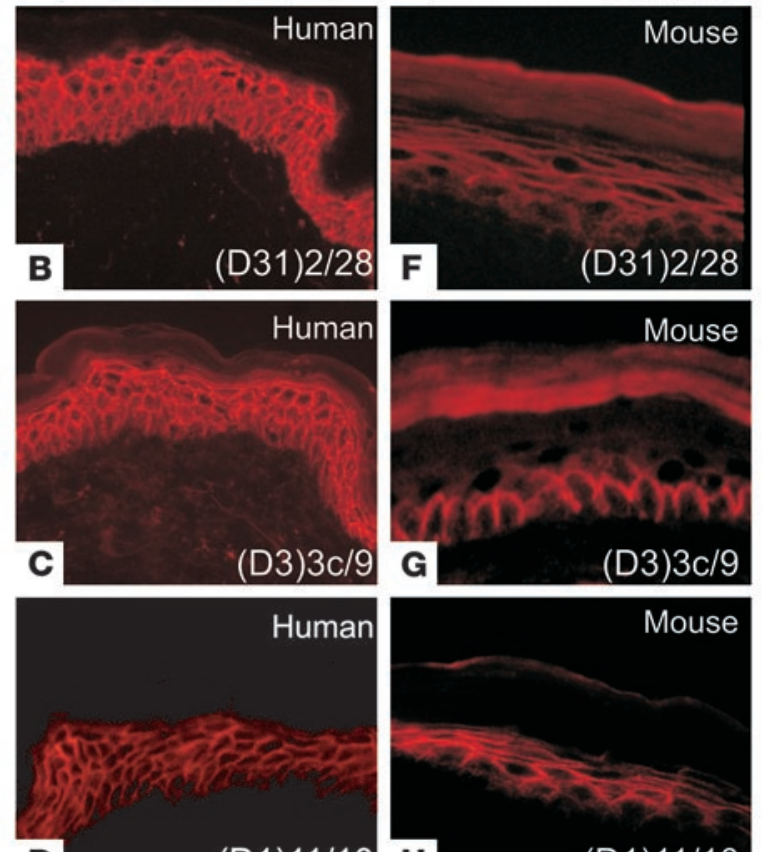

D

(D1) $11 / 10$
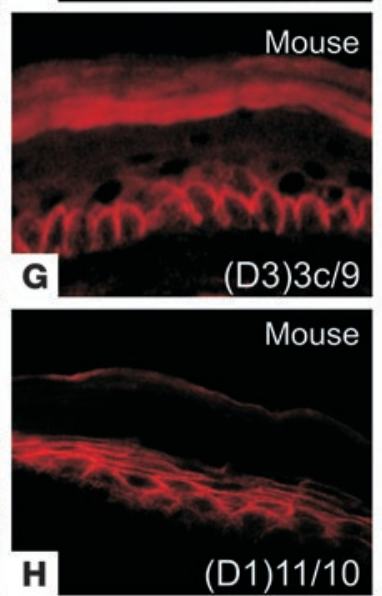

heavy chain $\left(\mathrm{V}_{\mathrm{H}}\right)$ and variable-region light chain $\left(\mathrm{V}_{\mathrm{L}}\right)$ fragments from peripheral blood lymphocytes isolated from a patient with active acute mucocutaneous PV. The resulting cDNA was then cloned into a phagemid vector, which facilitated the creation of an antibody phage display library, comprising approximately $4 \times 10^{8}$ independent transformants. Phage particles, each displaying a particular $V_{H}$ and $V_{L}$ pair on their surface (folded into a single monovalent antigen-binding site) with the corresponding cDNA encoding the pair within the particle, were selected by panning against the extracellular domain of desmoglein substrate immobilized on ELISA plates. We performed 4 rounds of panning with scFvs selected against only Dsg3 (D3), only Dsg1 (D1), or both Dsg3 and Dsg1 (D31). Phage clones that expressed desmoglein-specific antibodies were identified after the second through fourth rounds of panning and were subsequently confirmed by ELISA, using enzyme-conjugated anti-M13 phage antibody to detect antigen-binding phage particles. 26 D3 clones, 24 D1 clones, and 13 D31 clones were randomly selected for nucleotide sequencing (see Figure 1A for nomenclature). Of these, we identified 16, 22, and 5 unique mAbs, respectively, based on combinations of distinct heavy and light

\section{Figure 3}

Characterization of scFv mAb desmoglein-binding specificity by ELISA. (A) Patterns of the binding of selected scFv mAb clones to Dsg1 and Dsg3. (D3)3c/9 was isolated from a library panned on Dsg3 but showed quantifiable specificity for Dsg1, as shown in $\mathbf{B}$. Similar results were obtained for mAb (D3)3a/9 (data not shown).

\section{Figure 2}

IIF of scFv mAbs on human and mouse epidermis. The ScFv mAb used as primary antibody for staining human $(\mathbf{A}-\mathbf{E})$ and mouse $(\mathbf{F}-\mathbf{H})$ epidermis is indicated in each panel. Magnification, $\times 400$.

chain variable-region sequences. To perform functional studies for this cohort of mAbs, a nonsuppressor strain of E. coli was used to express the recombinant human mAbs as soluble scFv molecules unlinked to phages. Soluble scFvs, which were engineered with a 6x-histidine tag and an HA peptide tag on their carboxy termini, were purified by nickel-chelation chromatography.

The purity of the $\mathrm{scFv}$ preparation was evaluated by Coomassie staining after SDS-PAGE, which showed a single predominant protein band (Figure 1B). Gel-filtration HPLC indicated that soluble scFvs were $92-98 \%$ monomeric (Figure 1C). These data show that the scFvs can be easily purified and consist almost completely of monomers with little, if any, aggregation.

The repertoire of human $P V s c F v$ mAbs shows various patterns of desmoglein-specific binding as determined by indirect immunofluorescence and ELISA. ScFvs were tested by indirect immunofluorescence (IIF) on normal human skin using anti-HA antibodies for detection in order to evaluate the ability of recombinant mAbs to bind native desmogleins in human tissue. In general, scFvs derived from phage libraries panned on Dsg3 showed the expected binding to the keratinocyte cell surface in the basal and immediate suprabasal layers, where Dsg3 is expressed (Figure 2A), and those derived from libraries panned on Dsg3 and Dsg1 bound throughout the epidermis (Figure 2B). However, 2 clones panned only on Dsg3, (D3)3c/9 and (D3) $3 \mathrm{a} / 9$, bound throughout the epidermis (Figure $2 \mathrm{C}$ and data not shown). As discussed below, by ELISA these scFv mAbs showed not only binding to Dsg3 but also slight binding to Dsg1. Interestingly, only 1 of the $\mathrm{scFv}$ mAbs derived from a phage library selected on Dsg1 stained human epidermis by IIF, but that antibody bound in the expected, predominantly superficial, cell surface pattern (Figure 2D).
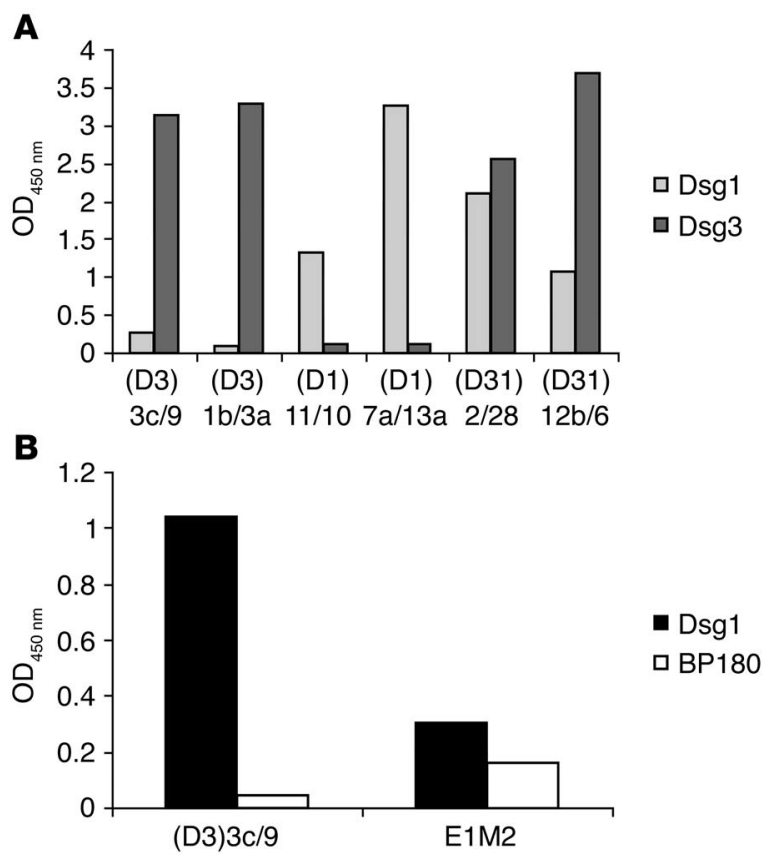


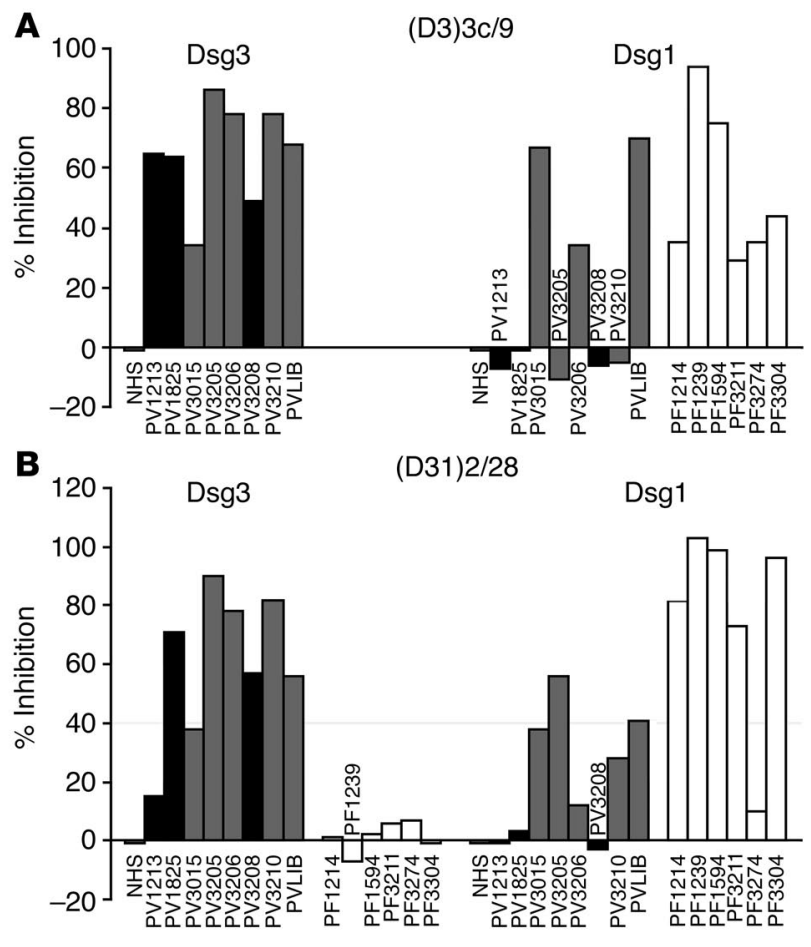

We then tested our cohort of recombinant $\mathrm{scFv}$ mAbs by ELISA to more precisely determine their desmoglein-binding specificities. In general, antibodies derived from libraries panned on Dsg3 bound Dsg3 but not Dsg1 [e.g., clone (D3)1b/3a; Figure 3A]. However, clones (D3)3c/9 and (D3)3a/9, although selected for binding against only Dsg3, also demonstrated weak binding to Dsg1 (Figure 3, A and B, and data not shown), consistent with their IIF binding throughout human epidermis (Figure 2C). This binding to Dsg1 was specific, as a higher concentration of (D3)3c/9 bound strongly to Dsg1 but not to an irrelevant autoantigen (bullous pemphigoid 180 [BP180] antigen), and an irrelevant ScFv (E1M2, a human red blood cell $\mathrm{mAb}$; ref. 25) did not bind Dsg1 when used at an equivalent $\mathrm{scFv}$ protein concentration (Figure $3 \mathrm{~B}$ ). As would be expected, scFvs from D1 clones bound Dsg1 by ELISA, while D31 clones that were derived from a phage library alternately panned on Dsg1 and Dsg3 bound both desmogleins (Figure 3A). These data demonstrate that we have successfully isolated antiDsg scFv mAbs from a patient with mucocutaneous PV, and that a single $\mathrm{mAb}$ can bind both Dsg3 and Dsg1. These recombinant antibodies reproduce binding of desmogleins by both ELISA and immunohistochemical assays.

In order to test for pathogenicity of selected $\mathrm{scFv} \mathrm{mAbs}$ in the neonatal mouse model of pemphigus, we first needed to demonstrate $\mathrm{scFv}$ binding to mouse epidermis by IIF. Five D31 mAbs demonstrated binding throughout mouse epidermis, with more superficial staining (Figure 2F). Interestingly, most of the D3 scFv mAbs did not bind to mouse epidermis. However, clone (D3)3c/9 did bind to mouse epidermis, but, unlike in human epidermis, in which it bound throughout, it bound predominantly to the cell surface in the basal layer (Figure $2 \mathrm{G}$ ), suggesting that, in mice, it only binds to Dsg3. Similar to findings with human epidermis, most D1 scFv mAbs did not bind mouse epidermis, with the exception of (D1)11/10, which bound throughout (Figure $2 \mathrm{H}$ ). Therefore, not all human autoantibodies bind to mouse skin, and some

\section{Figure 4}

Epitopes recognized by PV scFv mAbs are blocked by sera from multiple pemphigus patients. (A) (D3)3c/9. Left: Inhibition of Dsg3 binding by all $8 \mathrm{PV}$ sera. PVLIB is the plasma of the mucocutaneous PV patient from whom the phage display library was made. Black bars represent PV sera containing anti-Dsg3 antibodies only; gray bars indicate PV sera containing antibodies with both anti-Dsg3 and anti-Dsg1 activity $[\mathrm{PV}(3+1)$ sera]. Right: Inhibition of Dsg1 binding by 3 of $5 \mathrm{PV}(3+1)$ sera and 6 of 6 PF sera (white bars). (B) (D31)2/28. Left: Inhibition of Dsg3 binding by 7 of 8 PV sera and 0 of 6 PF sera. Right: Inhibition of Dsg1 binding by 4 of $5 \mathrm{PV}(3+1)$ sera and 5 of $6 \mathrm{PF}$ sera. Inhibition of greater than $20 \%$ was considered positive. NHS, normal human sera.

of those that do may bind in differing patterns. Thus, our results indicate that there may be limitations in interpreting pathogenicity (especially the lack of pathogenicity) of serum-derived polyclonal human antibodies in the neonatal mouse model.

Sera from $P V$ and PF patients block binding of $s c F v m A$ bs to desmogleins in inbibition ELISA studies. To determine whether sera from different pemphigus patients bind similar or identical epitopes to those defined by the $\mathrm{scFv} \mathrm{mAbs}$ cloned from our PV patient, inhibition ELISA assays were performed. PF sera containing anti-Dsg1 antibodies, PV sera containing anti-Dsg3 antibodies [PV(3) sera], or PV sera containing both anti-Dsg3 and anti-Dsg1 antibodies $[\mathrm{PV}(3+1)$ sera] were evaluated for their ability to inhibit binding of scFv mAbs to desmoglein substrates. Figure 4 shows representative inhibition ELISA studies with scFvs (D3)3c/9 (Figure 4A) and (D31)2/28 (Figure 4B). Inhibition greater than $20 \%$ was scored as positive. All 8 PV sera tested (including the PV patient sera used to make the phage display library [PVLIB]) inhibited binding of (D3)3c/9 to Dsg3 (Figure 4A, left). All 6 of the PF sera tested, 3 of 5 PV $(3+1)$ sera (including PVLIB), and none of the $3 \mathrm{PV}(3)$ sera inhibited

\section{Table 1}

Summary of inhibition ELISA studies

\begin{tabular}{lcccc} 
ScFv & \multicolumn{2}{c}{ PV sera } & \multicolumn{2}{c}{ PF sera } \\
& Dsg3 & Dsg1 & Dsg3 & Dsg1 \\
(D1)5b/16a & - & $4 / 4$ & - & $6 / 6$ \\
(D1)6b/14 & - & $1 / 5^{\mathrm{A}}$ & - & $6 / 6$ \\
(D1)7a/13a & - & $2 / 4$ & - & $6 / 6$ \\
(D1)8/12a & - & $1 / 5^{\mathrm{A}}$ & - & $6 / 6$ \\
(D1)11/10 & - & $0 / 5^{\mathrm{B}}$ & - & $0 / 6^{\mathrm{B}}$ \\
(D3)1b/3a & $7 / 8$ & - & - & - \\
(D3)1d/2c & $7 / 7$ & - & - & - \\
(D3)1g/2e & $7 / 7$ & - & - & - \\
(D3)1i/4d & $8 / 8$ & - & - & - \\
(D3)3a/9 & $8 / 8$ & $3 / 5$ & - & $6 / 6$ \\
(D3)3b/8 & $8 / 8$ & - & - & - \\
(D3)3c/9 & $8 / 8$ & $3 / 5$ & $0 / 2$ & $6 / 6$ \\
(D3)4/30 & $7 / 7$ & - & - & - \\
(D31)12a/5 & $8 / 8$ & $1 / 5^{\mathrm{A}}$ & $0 / 6$ & $6 / 6$ \\
(D31)12b/6 & $8 / 8$ & $1 / 5^{\mathrm{A}}$ & $0 / 6$ & $6 / 6$ \\
(D31)2/28 & $7 / 8$ & $4 / 5$ & $0 / 6$ & $5 / 6$ \\
(D31)2/29 & $7 / 7$ & $4 / 4$ & - & $6 / 6$ \\
\hline
\end{tabular}

AReactions in which sera were used at a standard dilution of 1:25 instead of 1:6.25. In these cases, lack of inhibition may reflect lower titers of blocking antibody during ELISA inhibition, since in inhibition studies of $\mathrm{PV}(3+1)$ sera against Dsg1-reactive scFvs, higher concentrations of sera resulted in higher levels of inhibition. ${ }^{B}$ Paradoxically, PF and $P V(3+1)$ sera potentiated binding of (D1)11/10 to Dsg1. -, not tested. 


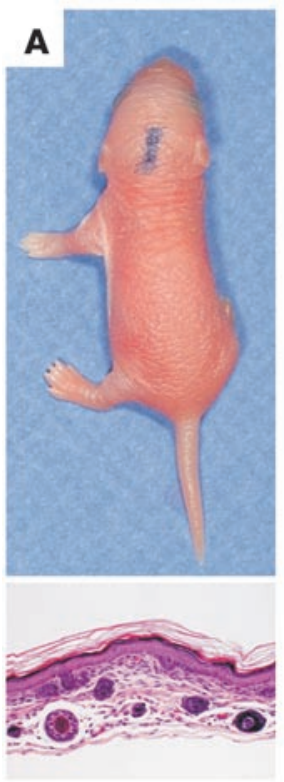

(D3) $3 c / 9$
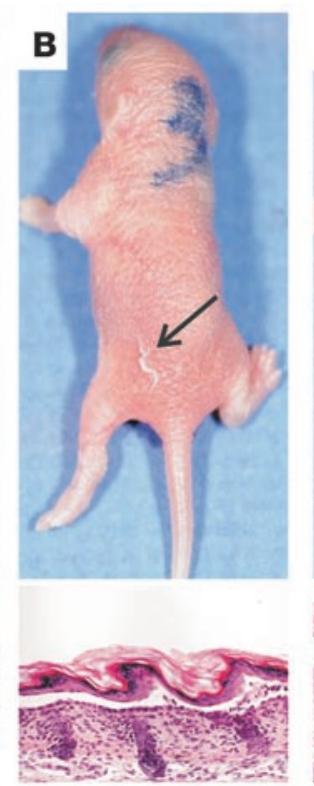

(D3) $3 \mathrm{c} / 9+\mathrm{ETA}$

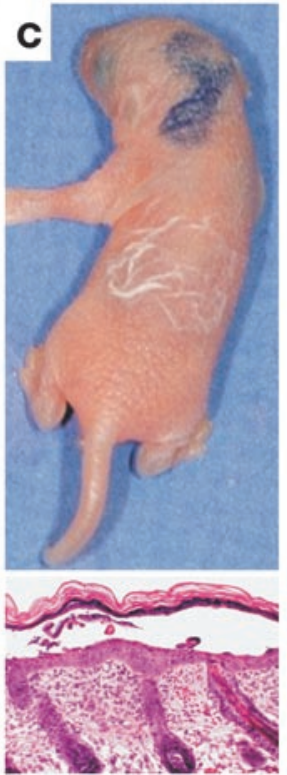

(D31)2/28

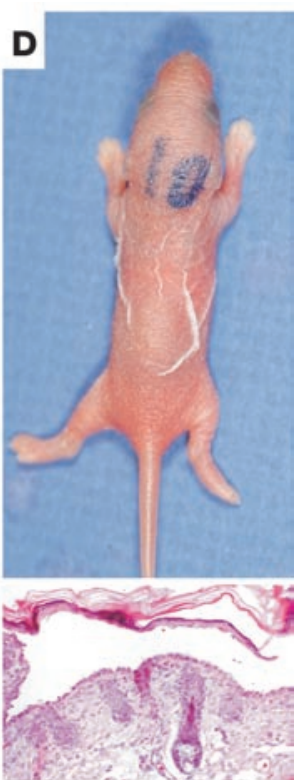

(D3) $3 \mathrm{c} / 9+(\mathrm{D} 31) 2 / 28$

\section{Figure 5}

Anti-Dsg scFv mAbs are pathogenic in neonatal mice. (A) Injection of (D3)3c/9 alone into neonatal mice does not cause gross blistering; histologic examination (lower panel) supports this result. (B) Injection of (D3)3c/9 with low-dose ETA demonstrates suprabasilar blistering. (C) Injection of (D31)2/28 alone demonstrates superficial blistering in the granular layer of the epidermis. (D) Coinjection of (D3)3c/9 and (D31)2/28 demonstrates suprabasilar blistering. Magnification, $\times 400$. binding of (D3)3c/9 to Dsg1 (Figure 4A, right). The $2 \mathrm{PV}(3+1)$ sera that did not inhibit (D3)3c/9 binding to Dsg1 demonstrated low (less than 1:20) ELISA titers against Dsg1, potentially accounting for the lack of inhibition. Seven of 8 PV sera (including PVLIB) and 0 of 6 PF sera inhibited (D31)2/28 binding to Dsg3 (Figure 4B, left). Five of 6 PF sera, 4 of 5 PV $(3+1)$ sera (including PVLIB), and 0 of $3 \mathrm{PV}(3)$ sera inhibited binding of (D31)2/28 to Dsg1 (Figure 4B, right). The number of $\mathrm{PV}$ patient- or $\mathrm{PF}$ patient-derived sera that blocked binding of each scFv to Dsg3 or Dsg1 was tabulated, with inhibition greater than $20 \%$ scored as positive (Table 1). D31 and D1 clones were inhibited from binding to Dsg1 by both PF sera and $\mathrm{PV}(3+1)$ sera, but not by normal human control or PV(3) sera, which indicates that the inhibition of $\mathrm{scFv}$ binding is due to the presence of anti-Dsg1 antibodies in pemphigus sera.

These data suggest that epitopes defined by the scFv mAbs derived from our mucocutaneous PV patient are also bound or blocked by sera from most pemphigus patients.

Phage display isolates both pathogenic and nonpathogenic scFv antibodies. $\mathrm{ScFv}$ polypeptides consist of a single monovalent variable-region antigen-binding site. To evaluate the ability of anti-Dsg scFvs to disrupt keratinocyte adhesion in vivo, scFvs that bound mouse skin by IIF were administered to neonatal mice by s.c. injection. To determine whether anti-Dsg3 scFvs were pathogenic, in some cases we coinjected low-dose exfoliative toxin A (ETA) to inactivate Dsg1 in the deep epidermis, since induction of typical PV suprabasilar blistering requires inactivation of both desmogleins. Injection of PBS, lowdose ETA alone, or (D3)3c/9 alone did not result in skin blistering of neonatal mice (Figure 5A and data not shown). However, concomitant injection of (D3)3c/9 with low-dose ETA to cleave Dsg1 caused blistering of the skin within 2-6 hours (Figure 5B). Indirect and direct immunofluorescence on mouse epidermis confirmed the presence of scFvs in the serum and skin of injected mice, with IIF titers ranging from $1: 1,600$ to $1: 4,000$. Histologic analysis of skin sections from mice injected with (D3)3c/9 and low-dose ETA demonstrated suprabasilar acantholysis characteristic of PV blisters (Figure 5B, lower panel). Subcutaneous injection of (D31)2/28 (without ETA) into neonatal mice resulted in blistering of the skin within 3 hours, with histology demonstrating superficial acantholysis similar to lesions seen in PF (Figure 5C). Clinical blistering was observed with IIF titers of mouse sera as low as 1:20. In contrast, injection of (D31)12a/5, (D31)12b/6, and (D31)12c/7 into neonatal mice did not result in skin blisters, despite IIF experiments detecting scFvs in mouse sera at titers ranging from 1:50 to 1:4,000 (data not shown).

Because the patterns of histologic blistering implied recognition of murine Dsg3 (mDsg3) by (D3)3c/9 and murine Dsg1 (mDsg1) by (D31)2/28, injection of both scFvs into neonatal mice was performed. IIF titers after scFv injection ranged from 1:400 to 1:4,000. Mice developed gross blisters within 2 hours, which on histologic examination demonstrated suprabasilar blistering identical to what is observed in lesions of mucocutaneous PV (Figure 5D). These data show that some, but not all, scFv monovalent antibodies are pathogenic in mice and presumably have the ability to disrupt adhesion by their target desmogleins.

However, given that anti-Dsg scFv binding patterns appear to be species-specific, mouse models may not allow for accurate evaluation of pathogenicity of human autoantibodies for

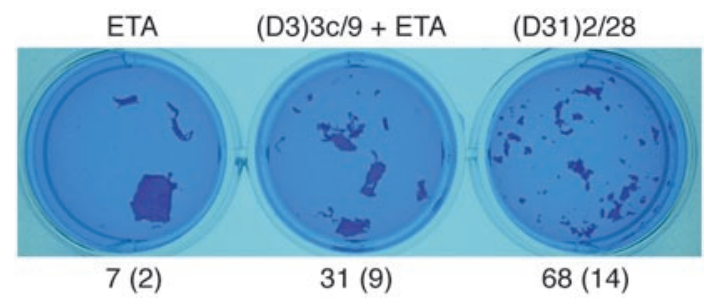

\section{Figure 6}

ScFv mAbs cause dissociation of cultured human epidermal keratinocytes. Cultured human keratinocytes were incubated with ETA with or without scFvs and then treated with dispase to release cell monolayers. Released cell sheets were exposed to mechanical shear stress to evaluate intercellular adhesion. The total number of cell sheet fragments for each treatment is shown as a mean (SD). 


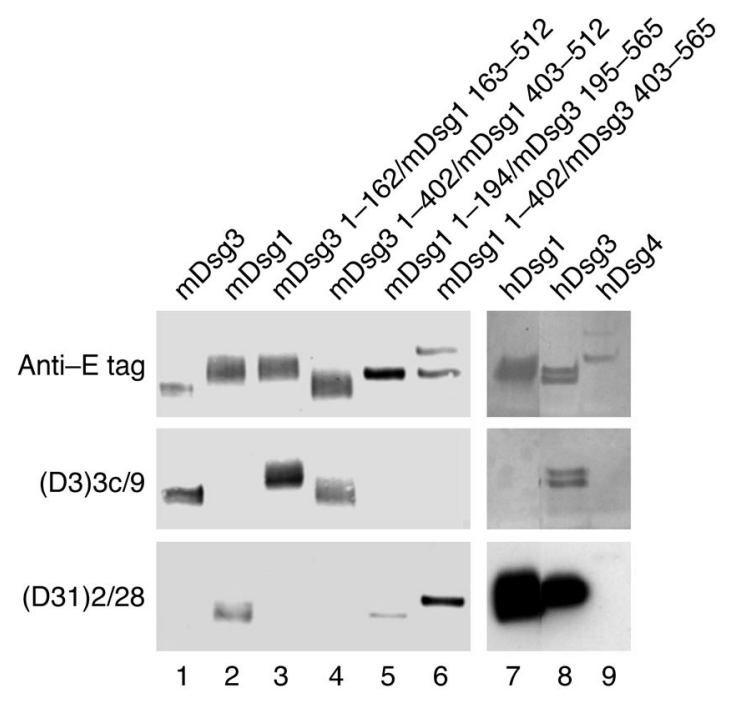

Figure 7

Epitope mapping of (D3)3c/9 and (D31)2/28 against mouse and human desmogleins. Wild-type and domain-swapped extracellular domains of $\mathrm{mDsg} 1$ and $\mathrm{mDsg} 3$ (lanes 1-6) or wild-type extracellular domains of human desmogleins (hDsgs) 1, 3, and 4 (lanes 7-9) were produced in baculovirus and immunoprecipitated with scFv mAbs. Desmoglein proteins bound by human scFv mAbs were detected by immunoblot analysis using antibody against $E$ tag, which was engineered onto the carboxyterminal domain of recombinant desmoglein molecules. The top panel shows the immunoblot of the recombinant and chimeric desmogleins detected with anti-E tag. The bottom 2 panels show the results of the immunoprecipitation-immunoblotting studies.

human desmogleins. To evaluate pathogenicity of scFvs against human keratinocytes, an assay for dissociation of cultured human keratinocytes was used (26). Cultured primary human epidermal keratinocytes express Dsg1 and Dsg3 with minimal levels of Dsg2 (26). Incubation of cultured keratinocytes with ETA alone did not cause significant dissociation (Figure 6, left). However, incubation of keratinocytes with (D3)3c/9 in the presence of ETA resulted in cell sheet dissociation (Figure 6, middle). (D31)2/28 was able to induce cell sheet dissociation in the absence of ETA (Figure 6, right). These data suggest that (D3)3c/9 can interfere with adhesion mediated by human Dsg3 and that (D31)2/28 can disrupt both human Dsg3 and human Dsg1. Incubation of cultured keratinocytes with (D3)1b/3a, (D3)1d/2c, (D3) $1 \mathrm{~g} / 2 \mathrm{e},(\mathrm{D} 3) 1 \mathrm{i} / 4 \mathrm{~d}$, (D31)12a/5, and (D31)12b/6 did not result in cell sheet dissociation, providing further evidence that both pathogenic and nonpathogenic pemphigus antibodies were isolated.

Epitope mapping on domain-swapped desmogleins shows that pathogenic epitopes defined by scFv $m A$ bs are on the amino termini of Dsg1 and Dsg3. The different binding patterns of scFvs on mouse and human epidermis, together with the neonatal mouse pathogenicity data, suggested that (D3)3c/9 recognized only $\mathrm{mDsg} 3$ and not $\mathrm{mDsg} 1$, and that (D31)2/28 recognized only mDsg1 and not mDsg3. To confirm these empirical observations and further delineate the site of $\mathrm{scFv}$ binding, epitope mapping of (D3)3c/9 and (D31)2/28 on domainswapped mouse desmogleins was performed. Figure 7 (lanes 1-6) shows that (D3)3c/9 binds an epitope on $\mathrm{mDsg} 3$ contained within the amino-terminal 162 amino acids. No binding of (D3)3c/9 to mDsg1 was detected. Conversely, (D31)2/28 recognized epitopes in the 194-amino acid amino-terminal domain of mDsg1 with no detectable binding to mDsg3. Therefore, consistent with previously published studies of polyclonal human sera and a pathogenic mouse anti-Dsg $\mathrm{mAb}$ (19-21), our pathogenic recombinant human scFvs bind epitopes on the amino termini of desmogleins.

The finding of such intense superficial staining of human epidermis with (D3)3c/9 (Figure 2C), which demonstrated only weak ELISA binding to Dsg1 (Figure 3B), led us to question whether this $\mathrm{scFv}$ might also bind Dsg4, which has previously been reported to be expressed in the superficial layers of interfollicular human epidermis (27). However, neither (D3)3c/9 nor (D31)2/28, which also stains superficial epidermis, bound to human Dsg4 (Figure 7, lane 9).

ScFv mAbs recognize primarily but not exclusively calcium-stabilized conformational epitopes on desmogleins. As most pathogenic pemphigus antibodies have been shown to recognize conformational epitopes on desmogleins that are stabilized by calcium $(28,29), \operatorname{scFv} m A b s$ were tested by IIF and/or ELISA on EDTA-treated substrate, as well as by immunoblot analysis, to determine whether they bound linear or conformational epitopes.

ScFvs from clones (D3)3c/9, (D3)1b/3a, and (D31)2/28 were tested by IIF on human epidermal sections pretreated with EDTA. Such pretreatment to chelate divalent cations (presumably calcium in the case of desmogleins) abrogated or markedly diminished binding (Figure 2, C versus E). To further evaluate this inhibition of binding, ELISA assays were performed for representative scFvs, using desmoglein substrates with and without EDTA pretreatment. EDTA inhibited binding of scFvs to varying degrees, with most demonstrating greater than $50 \%$ inhibition by EDTA (Table 2). However, 2 nonpathogenic antibodies, (D31)12a/5 and (D31) $12 \mathrm{~b} / 6$, demonstrated only $5-26 \%$ inhibition of binding to Dsg3 by EDTA, suggesting that the epitopes recognized by some scFvs do not require calcium for epitope stabilization.

ScFvs were also evaluated for binding to denatured desmogleins by immunoblot analysis of human keratinocyte extracts. None of $17 \mathrm{mAbs}$ recognized Dsg1, whereas the murine anti-Dsg1 $\mathrm{mAb}$ P124 did show the expected $160-\mathrm{kDa}$ band (data not shown). When tested at antibody concentrations greater than 100-fold higher than their ELISA titer for an OD of 1, 8 of 8 D3 mAbs and 3 of 3 D31 mAbs demonstrated specific binding to a $130-\mathrm{kDa}$ protein that comigrated with the band recognized by the murine anti-Dsg3 mAb 5G11 (Figure 8), suggesting either that some renaturation takes place after SDS-PAGE or that there is some affinity for denatured epitopes. These immunoblot data underscore the exquisite specificity of the scFv mAbs for Dsg3 and highlight their potential utility as laboratory detection reagents.

\section{Table 2}

EDTA-induced inhibition of scFv mAb binding to desmoglein substrates

\begin{tabular}{lcc} 
ScFv & \multicolumn{2}{c}{ \% Inhibition by EDTA [mean (SD)] } \\
& Dsg1 & Dsg3 \\
(D1)11/10 & $96(2)$ & - \\
(D3)1b/3a & - & $78(6)$ \\
(D3)3c/9 & - & $84(2)$ \\
(D31)2/28 & $65(5)$ & $90(2)$ \\
(D31)2/29 & $89(2)$ & $93(3)$ \\
(D31)12a/5 & $40(3)$ & $26(8)$ \\
(D31)12b/6 & $52(4)$ & $5(3)$
\end{tabular}

Values assessed by ELISA. 


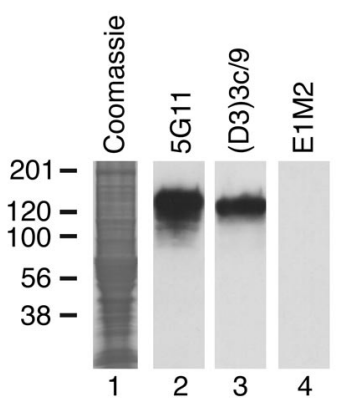

PV mAbs with different desmoglein-binding specificities demonstrate different patterns of heavy and light chain gene restriction. The third complementarity-determining region (CDR3) comprises the unique genetic sequence spanning the VDJ recombinatory junctions of the Ig heavy chain (reviewed in ref. 30). This region defines a particular $\mathrm{B}$ lymphocyte and all of its clonal progeny. To determine the genetic interrelatedness of antibodies captured by phage display, a total of $63 \mathrm{mAbs}$ (26 D3, 24 D1, and 13 D31) were randomly selected for

\section{Figure 8}

PV scFv mAbs recognize primarily but not exclusively conformational epitopes on desmogleins. Immunoblot analysis of human keratinocyte extract using (D3)3c/9. Lane 1, Coomassie blue-stained human keratinocyte extract. Lane 2, murine anti-Dsg3 mAb 5G11. Lane 3, (D3)3c/9. Lane 4, E1M2 (control anti-human red blood cell scFv).

nucleotide sequencing of the heavy and light chain variable regions. Several mAbs shared identical CDR3 sequences, indicating derivation from a common parental B cell clone. Twelve unique CDR3 sequences, designated as VDJ1-VDJ12, were identified among the 63 desmoglein-reactive clones. Figure 9A shows the CDR3 alignments for D3, D1, and D31 heavy chain sequences, together with the $\mathrm{V}_{\mathrm{H}}$ gene and gene family usage. Both the heavy and the light chain utilization for each unique anti-Dsg $\mathrm{mAb}$ is depicted in Figure 9B.

Of the $26 \mathrm{D} 3 \mathrm{mAbs}$, there were 13 unique heavy chain sequences represented, which could be grouped into 3 common VDJ families based on their CDR3 sequence, with each VDJ family representing a clonal population derived from a common $\mathrm{B}$ cell parental clone (Figure 9A, upper panel). Restriction of D and JH gene segments was
A

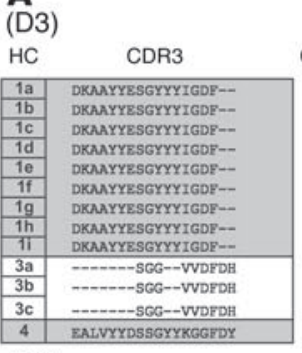

(D1)

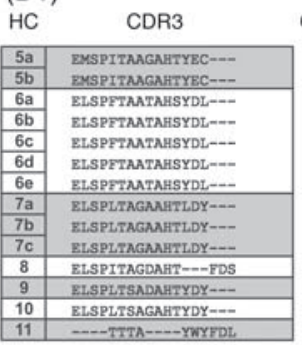

(D31)

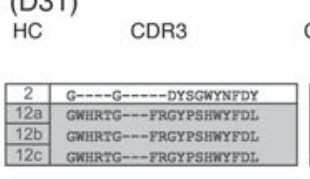

Common VDJ VH gene VH family

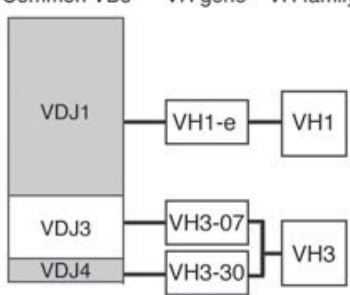

Common VDJ VH gene VH family

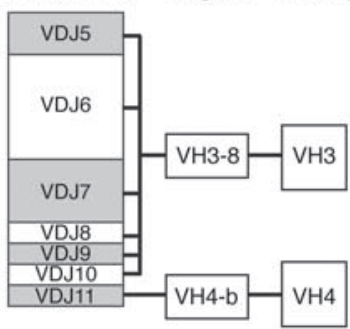

Common VDJ VH gene VH family

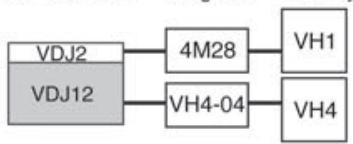

B

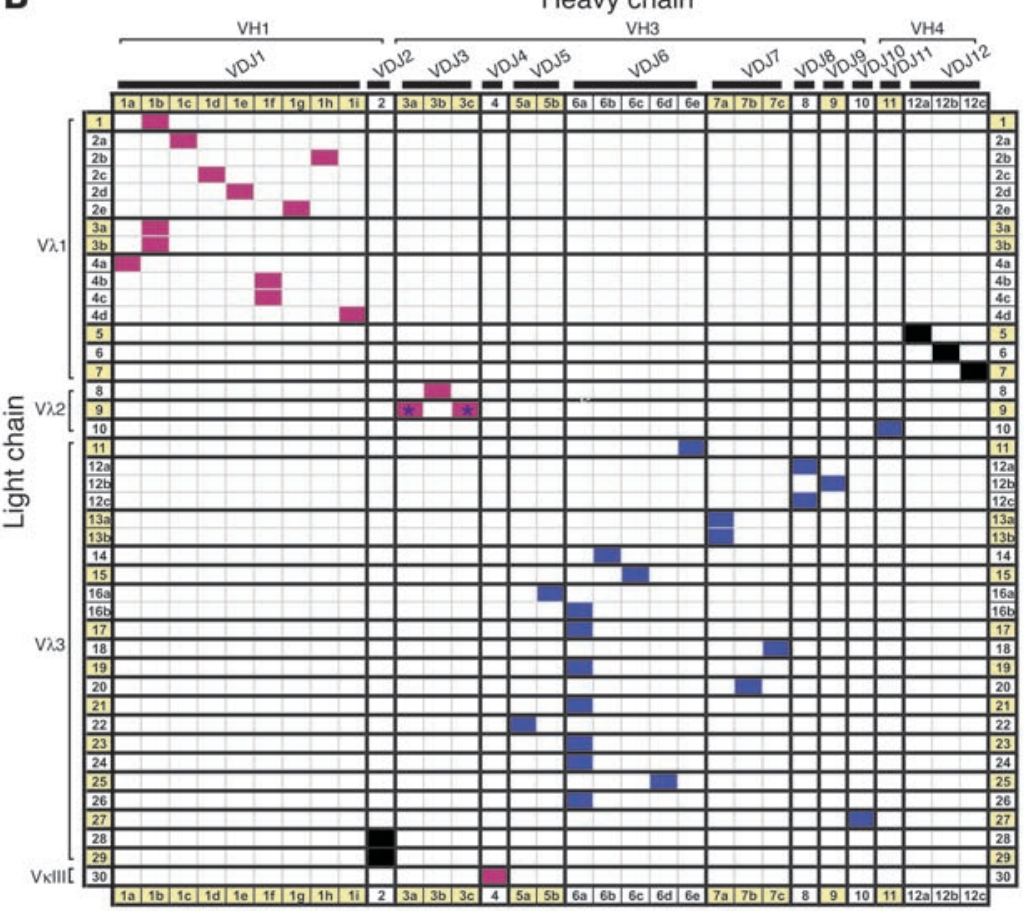

\section{Figure 9}

Heavy and light chain restriction of Dsg-panned scFv mAbs. (A) Dendrogram and CDR3 alignment of (D3), (D1), and (D31) heavy chain sequences. Heavy chain (HC) sequence analysis of 63 randomly selected mAbs from the PV autoantibody repertoire identified 12 different VDJ families (indicated as VDJ1-VDJ12). Each VDJ family shares a common B cell precursor, defined by a common CDR3 amino acid sequence. The presence of a letter suffix in the heavy chain indicates the presence of somatic mutations in the variable region outside the CDR3, which reflect genetic diversification of the original parental $B$ cell clone. The $V_{H}$ gene family and gene segment usage is indicated for each of the 31 unique heavy chain sequences. (B) Unique pemphigus mAbs show restricted and nonoverlapping usage of heavy and light chain gene segments. The horizontal axis represents unique heavy chains, and the vertical axis represents the unique $\lambda$ and $\kappa$ light chains (based on nucleic acid sequence). The heavy chains are restricted into 12 VDJ groupings, whereas the light chain repertoire comprises 30 groupings, defined by a unique light chain junctional region. Of the 26, 24, and 13 randomly screened D3, D1, and D31 mAbs, 16, 22, and 5 unique heavy and light chain combinations, respectively, were identified (represented within the matrix as magenta, blue, and black boxes, respectively). In most cases, antibodies panned against a particular desmoglein bound only that desmoglein substrate. However, 2 mAbs, (D3)3a/9 and (D3)3c/9, although panned only against Dsg3, also weakly bound Dsg1 (indicated by asterisks). 
also observed, with exclusive utilization of only $2 \mathrm{D}$ gene segments and JH4b for all D3 clones. D3 clones displayed preferential usage of the $\mathrm{VH} 1 / \mathrm{V} \lambda 1$ gene families (Figure 9B). However, clone (D3)3c/9, which demonstrates binding of Dsg1 in addition to Dsg3, used $\mathrm{VH} 3 / \mathrm{V} \lambda 2$. (D3)3a/9, with the same desmoglein-binding specificity as (D3) $3 c / 9$, had heavy and light chain gene usage identical to that of(D3)3c/9 but different heavy chain somatic mutations. These data suggest that heavy and light chain gene family usage may determine the binding specificity of autoantibodies against desmogleins.

The 24 D1 mAbs derived from 7 different parental B cell clones (identified as VDJ5-VDJ11) (Figure 9A, middle panel). All but 1 of the 14 unique D1 heavy chain sequences were encoded by the VH3-8 gene. D1 clones showed preferential usage of $\mathrm{VH} 3 / \mathrm{V} \lambda 3$ (Figure 9B), with the exception of clone (D1)11/10, which used $\mathrm{VH} 4 / \mathrm{V} \lambda 2$. Interestingly, (D1)11/10 was also unique among D1 clones in its binding of human epidermis by IIF.

Analysis of 13 D31 clones revealed 4 unique heavy chain sequences derived from 2 different VDJ rearrangements (Figure 9A, lower panel). D31 clones showed restricted but distinct $\mathrm{V}_{\mathrm{H}}(\mathrm{VH} 1$ and $\mathrm{VH} 4)$ and $\mathrm{V}_{\mathrm{L}}(\mathrm{V} \lambda 1$ and $\mathrm{V} \lambda 3$ ) gene family usage (Figure 9B).

Of the 26 D3, 24 D1, and 13 D31 sequenced clones, 16, 22, and 5 unique heavy and light chain combinations were identified, respectively. As shown in Figure 9B, D3, D1, and D31 clones demonstrated restricted and nonoverlapping usage of both heavy and light chain genes. In some cases, multiple antibodies of the same heavy and light chain gene family were identified, differing only by somatic mutation from germ-line $V_{H}$ and $V_{L}$ Ig genes (e.g., VDJ1 with light chain family 2). That these mutations are not the result of PCR errors in library construction but more likely reflect a somatic mutation process in vivo is supported by the fact that Taq polymerase under the conditions used in these studies is associated with less than 1 error per 9,000 nucleotides (31). PCR with such a low error rate could not account for the number of mutations detected in our clones (i.e., as many as 20 or more per $\mathrm{V}_{\mathrm{H}}$ or $\mathrm{V}_{\mathrm{L}}$, each comprising approximately $350 \mathrm{bp}$ ) or their position (concentrated in CDRs; see below).

Genetic evidence of antigen-driven somatic mutation against Dsg3. Previous studies investigating the ratio of replacement to silent mutations $(\mathrm{R} / \mathrm{S})$ in productive versus nonproductive VDJ rearrangements of the Ig heavy chain have found that the $\mathrm{R} / \mathrm{S}$ ratio in antibody framework regions (FRs) from productive rearrangements ranges from 1.4 to 2.1 (32). The $\mathrm{R} / \mathrm{S}$ ratio within the CDRs of productive rearrangements tends to be higher, with values ranging from 4.7 to 5.0. To ask whether the nucleotide sequences of our cohort of human anti-Dsg3 $\mathrm{mAbs}$ provide evidence of antigen-driven somatic mutation, the pattern of somatic mutations within the D3 VH1encoded clonal tree was examined, specifically in regard to the ratio of replacement to silent mutations within the FRs and CDRs of the heavy chains. Unique D3 VH1 clones defined by a common CDR3 were aligned by heavy chain nucleotide sequence and compared with the nucleotide sequence predicted by the germ-line $V_{H}$ gene from which they most likely arose. Mutations from germ-line sequence were tallied as to location (FR versus CDR) and type (replacement versus silent). The calculated FR R/S ratio ranged from 1.5 to 2.3, and the CDR R/S ratio ranged from 9.0 to 10.0 ; these results are consistent with an antigen-induced somatic hypermutation process.

\section{Discussion}

Using phage display, we have cloned a repertoire of human monoclonal anti-Dsg autoantibodies, both pathogenic and nonpathogenic, from a patient with active mucocutaneous PV. We have con- firmed that these mAbs bind to recombinant desmogleins by ELISA and native desmogleins in human tissue by immunofluorescence analysis. ScFv binding to desmogleins can be blocked by sera from multiple pemphigus patients. Furthermore, these mAbs are pathogenic against keratinocytes in both human cell culture and a neonatal mouse model. Taken together, these data suggest that the anti-Dsg recombinant $\mathrm{mAbs}$ isolated by phage display reproduce the biochemical, immunohistochemical, and functional properties of polyclonal PV IgG. Our data demonstrate, for the first time to our knowledge, that distinct clonal populations of antibody molecules derived from a PV autoimmune repertoire mediate binding to Dsg3 and Dsg1. Furthermore, isolation of D31 clones definitively demonstrates that a single $\mathrm{mAb}$ can bind, and pathologically inactivate, both desmogleins. Although previous studies have shown little cross-reactivity of polyclonal antibodies between Dsg3 and Dsg1 $(2,8)$, closer examination of the data suggests that there may be a minor population of cross-reacting antibodies. Our data suggest that such antibodies might contribute to pathogenesis in mucocutaneous PV.

ScFvs are monovalent polypeptides comprising a single antigenbinding domain, which is devoid of Fc effector function. The finding that $\mathrm{scFv} \mathrm{mAbs}$ can induce blisters in mice clinically and histologically similar to those observed in pemphigus patients and also interfere with the adhesive function of Dsg1 and Dsg3 in cultured human keratinocytes is consistent with direct disruption of desmoglein interactions by pemphigus autoantibodies, as shown in previous studies using serum-derived Fab' fragments $(17,18)$. Epitope mapping data using pemphigus IgG or pathogenic mAbs isolated from an adoptive transfer mouse model of pemphigus show that almost all pathogenic autoantibodies bind conformational epitopes in the desmoglein amino-terminal extracellular domain that is predicted to form the intercellular trans-adhesive interface $(20,21,33)$. Consistent with these studies, 2 pathogenic scFvs, (D3)3c/9 and (D31)2/28, were shown to bind the amino terminus of desmogleins. Together with the ELISA EDTA inhibition (Table 2) and immunoblot findings, these data suggest that pathogenic pemphigus mAbs bind, at least partially, to calcium-stabilized epitopes in the amino termini of desmogleins, thereby directly inhibiting intercellular adhesion. These findings also provide further evidence that our recombinant pemphigus mAbs share biochemical mechanisms of pathogenicity with naturally occurring pemphigus autoantibodies.

With respect to the fine specificity and functional properties of anti-Dsg autoantibodies, our ability to isolate and characterize human mAbs has provided insight into the immunobiology of PV, which is difficult to discern with studies of polyclonal patient sera. $\mathrm{ScFv}$ mAbs bind to human epidermis with immunofluorescence patterns that are mostly consistent with their desmoglein-binding patterns by ELISA. However, IIF experiments demonstrated discrepancies in the binding of D1 scFvs to Dsg1 on ELISA and in tissue. The Dsg1 ELISA evaluates binding to the recombinant extracellular domain of Dsg1; thus it is possible that some epitopes on solid-phase immobilized substrate may either be more accessible or be configured differently from the native form in tissue. Additionally, we observed differential binding of scFvs to mouse and human epidermis by IIF. These immunofluorescence findings, confirmed by ELISA and immunoprecipitation mapping experiments on human and mouse desmoglein substrates, were consistent with pathogenicity studies. (D31)2/28 recognized both human Dsg1 and human Dsg3, consistent with its ability to induce dissociation of cultured human keratinocytes. In con- 
trast, (D31)2/28 bound only mDsg1, which explains the superficial site of blister formation in neonatal mice after $\mathrm{scFv}$ injection. In the case of (D3)3c/9, binding to human Dsg1 was detected by ELISA and IIF but not by immunoprecipitation (Figure 7, lane 7). The weak ELISA binding was likely specific, since this binding was inhibited by 6 of 6 PF sera and 3 of 5 PV $(3+1)$ sera. However, recognition of human Dsg1 by (D3)3c/9 is not likely to be pathophysiologically significant, as (D3)3c/9 was not pathogenic against Dsg1 in cultured human keratinocytes. These data underscore the importance of delineating the fine specificities of mAbs in order to explain their pathogenic activity, and they also highlight the difficulties of performing these more detailed evaluations of human autoantibodies within a mouse model. Studies using human keratinocyte substrates, either cultured or engrafted, may ultimately provide a more accurate assay for human autoantibody pathogenicity.

To further define the epitopes that are bound by pemphigus mAbs, inhibition ELISA assays with pemphigus patients' sera were performed. These experiments suggest that the epitopes bound by the mAbs derived from our patient are similar or identical to those recognized by other pemphigus patients' sera, for both pathogenic and nonpathogenic antibodies. Furthermore, $\mathrm{scFv} \mathrm{mAbs}$ from $\mathrm{D} 1$ clones were inhibited by sera from patients with both mucocutaneous PV and PF, suggesting that anti-Dsg1 antibodies from PV patients recognize similar epitopes to those recognized by $\mathrm{PF}$ antibodies generated during a primary autoimmune response to Dsg1. These data suggest that patterns of epitope recognition may be conserved between PF and PV patients. Although these scFvs were isolated from a single patient, the ELISA inhibition data indicate that the recombinant mAbs are biochemically representative of autoantibodies from multiple pemphigus patients in regard to immunologic epitopes.

Genetic analysis of pemphigus mAbs reveals both heavy and light chain gene restriction, with mAbs of different heavy and light chain families demonstrating different antigen-binding characteristics. The human $\operatorname{Ig} \mathrm{V}_{\mathrm{H}}$ repertoire consists of approximately 50 functional gene segments, which are divided into 7 gene families (VH1-VH7) based on nucleic acid identity (reviewed in refs. 30, 34). The majority of $\mathrm{V}_{\mathrm{H}}$ genes fall into the $\mathrm{VH} 3$ gene family (22 members, or 43\%), with $\mathrm{VH} 1, \mathrm{VH} 3$, and $\mathrm{VH} 4$ gene families collectively comprising $86 \%$ of all $\mathrm{V}_{\mathrm{H}}$ gene sequences. Restricted patterns of heavy and light chain gene usage have been described for a number of autoimmune and naturally occurring immune responses, including idiopathic thrombocytopenic purpura, systemic lupus erythematosus, rheumatoid arthritis, Sjögren syndrome, and Haemophilus influenzae type b infection, among others (35-39). However, nonstochastic utilization of $V_{H}$ gene segments has been reported to occur in the normal human antibody repertoire as well, with overrepresentation of $\mathrm{VH} 3-23$ and $\mathrm{VH} 4-34$, possibly due to mechanisms of both preferential rearrangement of $V_{\mathrm{H}}$ gene loci and receptor-dependent selection $(40,41)$. Our data demonstrate restricted patterns of $\mathrm{V}_{\mathrm{H}}$ gene usage for pemphigus antibodies, with preferential usage of VH1 family genes (notably VH1-e) and VH3 family genes for D3 clones, and VH3 family genes for D1 clones. D31 cloned antibodies that bound both Dsg3 and Dsg1 used both VH1 and VH4 gene segments.

The populations of $\mathrm{scFv}$ mAbs displaying varying desmogleinbinding specificities were classified into groups derived from common B cell precursors by virtue of their distinct CDR3 signatures (indicated by the VDJ rearrangement number in Figure 9B). These findings indicate that autoantibodies that bind Dsg3, Dsg1, or both derive from different parental B cell clones. Although R/S mutational analysis demonstrated genetic evidence of antigen-driven somatic mutation in D3 clones, we did not find any examples in which somatic mutation of anti-Dsg mAbs changed desmoglein-binding specificity (i.e., epitope migration; ref. 25). A significant implication of these data is that autoimmunity to Dsg1 likely does not derive from somatic hypermutation of antibody molecules reactive to Dsg3, and vice versa. Instead, our evidence shows that antibodies against Dsg3, compared with those that bind Dsg1 or both Dsg3 and Dsg1, come from genetically distinct $B$ cell populations.

The finding of a predominant (although not exclusive) use of $\lambda$ light chain sequences was unexpected, as pemphigus sera (including the one used to construct this library) contain autoantibodies comprising both $\lambda$ and $\kappa$ light chains (data not shown). This $\lambda$ predominance may reflect an actual increase in efficiency of binding of anti-Dsg antibodies with $\lambda$ chains in vivo or alternatively may reflect a selection bias during the panning of antibodies on ELISA plates. The lack of a specific light chain gene segment restriction in the D1 scFvs suggests that binding to Dsg1 is determined primarily by the heavy chain. Further studies, such as light chain shuffling experiments $(35,42)$ and screening of separate $\lambda$ and $\kappa$ phage libraries, will be necessary to fully delineate the light and heavy chain contributions to binding of desmogleins.

The above observations regarding $\mathrm{V}_{\mathrm{H}}$ gene segment restriction in anti-Dsg antibodies and possible shared epitopes among patients suggest several approaches that might be taken for novel, and more targeted, therapy of pemphigus patients. For example, in mice, infusions of microgram amounts of the B cell superantigen Staphylococcal protein A have been shown to cause apoptosis of B lymphocytes expressing antibodies of clan III gene-encoded $\mathrm{V}_{\mathrm{H}}$ segments, the murine homologs for human $\mathrm{VH} 3$ genes $(43,44)$. If certain $\mathrm{V}_{\mathrm{H}}$ gene segments predominate in pathogenic antibodies, then therapy could be directed against $B$ cells that express those segments. Additionally, if pathogenic $\mathrm{mAbs}$ define shared epitopes among patients, then these antibodies could be used to screen peptide phage display libraries for molecules that structurally mimic those epitopes. Such molecules could be used to block pathogenic antibodies in disease, an approach that has previously been successful for the inhibition of factor VIII autoantibodies in hemophilia (45). Furthermore, anti-idiotypic antibodies specific for autoantibodies from a pemphigus patient have shown promising results in blocking pathogenicity of autoantibodies from other patients (46). Our data showing that pathogenic mAbs define shared epitopes among patients are also consistent with the idea that the pathogenic antibodies share idiotypes. In this regard, scFvs could be used to raise specific anti-idiotype antibodies that in turn could be used to block pathogenic idiotypes in disease.

Finally, the phage display technique used here could also be useful for isolating antibodies against previously proposed nondesmoglein pemphigus antigens (47) to evaluate whether they are significant contributors to the pathophysiology of pemphigus.

We believe that, taken together, these studies describe the first successful cloning and characterization of pathogenic human monoclonal PV autoantibodies. These mAbs reproduce disease in both animal and human keratinocyte models and offer exciting opportunities for the development of novel biologic and clinical reagents to study, diagnose, and treat this potentially fatal disease. 


\section{Methods}

\section{Phage display}

Library construction. All human and animal studies were approved by the Institutional Review Board of the University of Pennsylvania. Using previously described methods (protocol 9.2, ref. 48), separate IgGK and IgG $\lambda$ phage libraries were constructed using the phagemid vector pComb3X (Scripps Research Institute) from $4 \times 10^{7}$ mononuclear cells isolated from $25 \mathrm{ml}$ of peripheral blood from a patient with active acute mucocutaneous PV. The phagemid library was electroporated into the XL1-Blue suppressor strain of E. coli (Stratagene) together with VCSM13 helper phages (Stratagene). Recombinant phages were purified from culture supernatants by polyethylene glycol precipitation and resuspended in PBS, pH 7.4, containing 1\% BSA.

The diversity of the unselected library was evaluated by sequencing of 42 randomly selected clones to determine the usage of $V_{H}$ and $V_{L}$ gene segments. Recombinant phagemids were purified with a plasmid preparation system (QIAGEN Inc.), and the $\mathrm{V}_{\mathrm{H}}$ and $\mathrm{V}_{\mathrm{L}}$ inserts were sequenced using pComb3X-specific primers previously described (protocol 11.24, ref. 48). Sequences were analyzed for homology to known human $\mathrm{V}, \mathrm{D}$, and J genes using the V BASE database (49).

Library selection. IgGK and IgG $\lambda$ antibody phage display libraries were combined in equal amounts and panned by solid-phase selection on ELISA plates coated with Dsg3 or Dsg1 (RhiGene Inc.). Briefly, freshly amplified phage library (approximately $1 \times 10^{11}$ to $5 \times 10^{11}$ phages) was incubated for 2 hours at $37^{\circ} \mathrm{C}$ on ELISA plate wells in PBS plus 2 $\mathrm{mM} \mathrm{CaCl}_{2}$. After washing of wells with $0.1 \%$ (vol/vol) Tween-20 in PBS plus calcium, adherent phages were eluted with $78 \mathrm{mM}$ citrate buffer, $\mathrm{pH}$ 2.4. After neutralization with $2 \mathrm{M}$ Tris, $\mathrm{pH} 7.4$, eluted phages were used to transform a fresh culture of XL1-Blue E. coli, which was amplified overnight as previously described (50). Phages were harvested from culture supernatants and then repanned against desmoglein substrate for 3 subsequent rounds as described for the original library. Individual phage clones were isolated from each round of panning, and binding to Dsg1 and Dsg3 was confirmed by ELISA using HRP-conjugated antiM13 antibody as the developing reagent (Roche Diagnostics Corp.). The heavy and light chain variable-region nucleic acid sequences were determined for positive clones in order to establish our cohort of unique anti-Dsg-specific mAbs.

\section{Production of soluble scFvs}

In order to produce $\mathrm{scFv}$ monoclonal preparations unlinked to phages, the TOP10F' nonsuppressor strain of E. coli (Invitrogen Corp.) was infected with an individual phage clone (protocol 11.6, ref. 48). Soluble scFv molecules were then purified from the bacterial periplasmic space using sucrose shock and nickel-chelation affinity chromatography as previously described (25). ScFvs were dialyzed against PBS and concentrated by Amicon ultrafiltration (Millipore Corp.) for some experiments. Various preparations of scFvs were normalized for approximate $\mathrm{scFv}$ concentrations by SDS-PAGE at different dilutions followed by immunoblotting with HRP-conjugated anti-HA antibodies (Roche Diagnostics Corp.).

\section{Gel-filtration HPLC}

Size exclusion chromatography was performed on a $30-\mathrm{cm} \times 8-\mathrm{mm}$ G2000SW TSK-GEL column (Tosoh Bioscience LLC) equilibrated with $0.15 \mathrm{M} \mathrm{NaCl}, 0.01 \mathrm{M}$ Mops, pH 6.8. Bovine transferrin (75 kDa; SigmaAldrich) and bovine erythrocyte carbonic anhydrase $(30 \mathrm{kDa}$; SigmaAldrich), $50 \mu \mathrm{g} / \mathrm{ml}$ in equilibration buffer, served as protein standards. 0.1 M Mops solution was added to all solutions as a marker of the total volume of the column. Approximately $5 \mu \mathrm{g}$ of scFvs $(200 \mu \mathrm{g} / \mathrm{ml}$ in PBS, pH 7.4) was injected into the column, eluted at $0.5 \mathrm{ml} / \mathrm{min}$, and detected by $\mathrm{OD}_{230 \mathrm{~nm}}$ absorbance using a PerkinElmer Inc. LC235 diode array detector. Data were analyzed with IGOR Pro software (WaveMetrics).

\section{ELISA binding and inbibition studies}

$\mathrm{ScFv}$ preparations were diluted in sample diluent (RhiGene Inc.) and incubated on Dsg3 and Dsg1 ELISA plates according to the manufacturer's directions for use with human sera. After washing, plates were developed with HRP-conjugated anti-HA antibody (Roche Diagnostics Corp.) and tetramethylbenzidine substrate. Absorbance was read at $450 \mathrm{~nm}$. Irrelevant scFvs (E1M2, a human anti-red blood cell antibody [ref. 25]; and AM3-13 [D.L. Siegel, unpublished observations]) were used at similar concentrations as negative controls. For some experiments, ELISA plate wells coated with the BP180 antigen substrate (RhiGene Inc.) were also used as negative controls.

Inhibition ELISA was performed by incubation of pemphigus or normal control sera (1:6.25 to 1:25) with scFvs on desmoglein ELISA plates. ScFvs were used at dilutions that result in OD readings (in the absence of putative blocking serum) of approximately 0.4 to 2.0 (linear range in plot of OD versus negative log of dilution). Inhibition was calculated according to the following formula:

\section{Equation 1 \\ $\%$ inhibition $=\left(1-\frac{O D_{S / B}-O D_{S C / B}}{O D_{S / B C}-O D_{S C / B C}}\right) \times 100$,}

where $S$ is the scFv being tested, $S c$ is $\mathrm{scFv}$ negative control, $B$ is blocking pemphigus serum, and $B c$ is normal human serum.

\section{Direct and indirect immunofluorescence}

Immunofluorescence using scFvs was performed on mouse tail, neonatal mouse skin, and human skin as previously described $(9,11,51,52)$ except for the detecting reagent, which comprised rat monoclonal anti-HA antibody (3F10; Roche Diagnostics Corp.) followed by Alexa Fluor 568-conjugated anti-rat IgG (Invitrogen Corp.).

\section{Immunoblot}

Primary human keratinocytes were cultured in defined keratinocyteserum-free media (keratinocyte-SFM; Invitrogen Corp.) supplemented with $1.2 \mathrm{mM}$ calcium chloride for 24 hours before harvesting. Cells were lysed with Laemmli sample buffer, and extracts were separated on denaturing SDS-PAGE and either stained with Coomassie reagent or transferred to nitrocellulose. Membranes were incubated with human scFvs or murine anti-Dsg3 mAb 5G11 (Zymed Laboratories Inc.) or anti-Dsg1 mAb P124 (Research Diagnostics Inc.) diluted in PBS $/ 5 \%$ milk. Blots were washed with PBS containing $0.1 \%$ (wt/vol) Tween-20 and then incubated with either HRP-conjugated anti-HA antibodies or HRP-conjugated goat antimouse antibodies (Bio-Rad Laboratories) diluted in PBS/5\% milk. Blots were developed using ECL Plus reagent (Amersham Biosciences Corp.).

\section{Neonatal mouse injection}

Injection of scFvs into neonatal mice was performed as previously described $(9,11,53)$. In some experiments, ETA was also injected into neonatal mice to cleave Dsg1 to determine whether anti-Dsg3 scFvs were pathogenic (21). Recombinant ETA with a $6 \mathrm{x}$-histidine tag was produced and purified on Ni-NTA columns (QIAGEN Inc.) as previously described (54). In preliminary experiments, neonatal mice were injected with various doses $(0.5-10 \mu \mathrm{g})$ of ETA to determine a dose that did not cause blistering alone (approximately $1 \mu \mathrm{g}$ ). ScFvs (approximately $20-100 \mu \mathrm{g}$ ) diluted in PBS were injected s.c. along the back into 1- to 2-day-old neonatal C57BL/6J 
mice weighing 1.2-1.8 g, with or without concomitant injection of ETA. Mice were sacrificed at 6-8 hours, 10 minutes after another injection with 25 units of heparin (Sigma-Aldrich). Skin was harvested for direct immunofluorescence by freezing in OCT (Electron Microscopy Sciences), and for histology by fixation in 10\% PBS-buffered formalin (SigmaAldrich). Blood was collected, and the plasma was used for IIF on normal mouse tail to determine the titer of circulating scFvs for each injection.

\section{Epitope mapping of scFvs against domain-swapped mouse desmoglein produced in baculovirus}

Domain-swapped desmoglein molecules comprising the extracellular domain of Dsg1 and Dsg3 were produced in baculovirus as previously described (33). ScFvs were incubated with $300 \mu \mathrm{l}$ of baculovirus culture supernatant containing the recombinant proteins for 30 minutes at room temperature. The proteins were immunoprecipitated with anti-HA agarose (Sigma-Aldrich) at $4{ }^{\circ} \mathrm{C}$ overnight and separated by SDS-PAGE. After transfer to polyvinyl difluoride membrane (Millipore Corp.), immunoblots were probed with antibody against $\mathrm{E}$ tag (Amersham Biosciences Corp.), which was engineered at the carboxy terminus of all recombinant desmoglein molecules.

\section{Dispase assay of cultured keratinocytes}

Primary human keratinocytes were seeded onto 12 -well plates in defined keratinocyte-SFM (Invitrogen Corp.) containing less than $0.1 \mathrm{mM}$ calcium. After reaching confluence, cells were incubated for 6 hours with scFvs at varying dilutions in defined keratinocyte-SFM supplemented with $0.4 \mathrm{mM}$ calcium chloride. For scFvs reactive to Dsg3 only, cells were also incubated with $0.5 \mu \mathrm{g} / \mathrm{ml}$ exfoliative toxin for the last 2 hours to inactivate Dsg1. Cultures were washed twice with PBS and then incubated with 1.2 units of dispase (Roche Diagnostics Corp.) for 30 minutes at $37^{\circ} \mathrm{C}$. Released monolayers were washed twice with PBS and gently pipetted through a 1-ml pipette tip 5 times to mechanically dissociate nonadherent cells. Cell sheet fragments were stained with crystal violet (Sigma-Aldrich) and quantitated with IPLab software (Scanalytics Inc.).

\section{Acknowledgments}

We would like to acknowledge John Seykora for discussions leading to the use of phage display in pemphigus and Norman Schechter for guidance with gel-filtration HPLC. This work was supported by National Research Service Award training grant T32-AR07465 (to A.S. Payne) and NIH grant R01 AR48223 (to J.R. Stanley). This work was also partly supported by Grants-in-Aid for Scientific Research from the Ministry of Education, Culture, Sports, Science, and Technology of Japan (to M. Amagai).

Received for publication December 14, 2004, and accepted in revised form February 8, 2005.

Address correspondence to: Aimee S. Payne, University of Pennsylvania, Department of Dermatology, Stellar Chance M8, 422 Curie Boulevard, Philadelphia, Pennsylvania 19104, USA. Phone: (215) 898-1818; Fax: (215) 573-9102; E-mail: aimee.payne@uphs.upenn.edu.
1. Payne, A.S., Hanakawa, Y., Amagai, M., and Stanley, J.R. 2004. Desmosomes and disease: pemphigus and bullous impetigo. Curr. Opin. Cell Biol. 16:536-543.

2. Ishii, K., et al. 1997. Characterization of autoantibodies in pemphigus using antigen-specific enzyme-linked immunosorbent assays with baculovirus-expressed recombinant desmogleins. J. Immunol. 159:2010-2017.

3. Ding, X., et al. 1997. Mucosal and mucocutaneous (generalized) pemphigus vulgaris show distinct autoantibody profiles. J. Invest. Dermatol. 109:592-596.

4. Amagai, M., Tsunoda, K., Zillikens, D., Nagai, T., and Nishikawa, T. 1999. The clinical phenotype of pemphigus is defined by the anti-desmoglein autoantibody profile. J. Am. Acad. Dermatol. 40:167-170.

5. Miyagawa, S., et al. 1999. Late development of antidesmoglein 1 antibodies in pemphigus vulgaris: correlation with disease progression. Br. J. Dermatol. 141:1084-1087.

6. Amagai, M., Hashimoto, T., Shimizu, N., and Nishikawa, T. 1994. Absorption of pathogenic autoantibodies by the extracellular domain of pemphigus vulgaris antigen (Dsg3) produced by baculovirus. J. Clin. Invest. 94:59-67.

7. Amagai, M., Hashimoto, T., Green, K.J., Shimizu, N., and Nishikawa, T. 1995. Antigen-specific immunoabsorption of pathogenic autoantibodies in pemphigus foliaceus. J. Invest. Dermatol. 104:895-901.

8. Ding, X., Diaz, L.A., Fairley, J.A., Giudice, G.J., and Liu, Z. 1999. The anti-desmoglein 1 autoantibodies in pemphigus vulgaris sera are pathogenic. J. Invest. Dermatol. 112:739-743.

9. Wu, H., et al. 2000. Protection of neonates against pemphigus foliaceus by desmoglein 3. N. Engl. J. Med. 343:31-35.

10. Shirakata, Y., Amagai, M., Hanakawa, Y., Nishikawa, T., and Hashimoto, K. 1998. Lack of mucosal involvement in pemphigus foliaceus may be due to low expression of desmoglein 1. J. Invest. Dermatol. 110:76-78.
11. Mahoney, M.G., et al. 1999. Explanation for the clinical and microscopic localization of lesions in pemphigus foliaceus and vulgaris. J. Clin. Invest. 103:461-468.

12. Wucherpfennig, K.W., et al. 1995. Structural basis for major histocompatibility complex (MHC)linked susceptibility to autoimmunity: charged residues of a single MHC binding pocket confer selective presentation of self-peptides in pemphigus vulgaris. Proc. Natl. Acad. Sci. U. S. A. 92:11935-11939

13. Hacker-Foegen, M.K., Fairley, J.A., and Lin, M.S. 2003. T cell receptor gene usage in desmoglein-3-specific $\mathrm{T}$ lymphocytes from patients with pemphigus vulgaris. J. Invest. Dermatol. 121:1365-1372.

14. Lin, M.S., et al. 1997. Development and characterization of desmoglein-3 specific $T$ cells from patients with pemphigus vulgaris. J. Clin. Invest. 99:31-40.

15. Veldman, C., Höhne, A., Dieckmann, D., Schuler, G., and Hertl, M. 2004. Type I regulatory T cells specific for desmoglein 3 are more frequently detected in healthy individuals than in patients with pemphigus vulgaris. J. Immunol. 172:6468-6475.

16. Nishifuji, K., Amagai, M., Kuwana, M., Iwasaki, T., and Nishikawa, T. 2000. Detection of antigen-specific $B$ cells in patients with pemphigus vulgaris by enzyme-linked immunospot assay: requirement of $\mathrm{T}$ cell collaboration for autoantibody production. J. Invest. Dermatol. 114:88-94.

17. Rock, B., Labib, R.S., and Diaz, L.A. 1990. Monovalent Fab' immunoglobulin fragments from endemic pemphigus foliaceus autoantibodies reproduce the human disease in neonatal Balb/c mice. J. Clin. Invest. 85:296-299.

18. Mascaro, J.M., Jr., et al. 1997. Mechanisms of acantholysis in pemphigus vulgaris: role of IgG valence. Clin. Immunol. Immunopathol. 85:90-96.

19. Sekiguchi, M., et al. 2001. Dominant autoimmune epitopes recognized by pemphigus antibodies map to the $\mathrm{N}$-terminal adhesive region of desmogleins. J. Immunol. 167:5439-5448.

20. Li, N., Aoki, V., Hans-Filho, G., Rivitti, E.A., and Diaz, L.A. 2003. The role of intramolecular epitope spreading in the pathogenesis of endemic pemphigus foliaceus (fogo selvagem). J. Exp. Med. 197:1501-1510.

21. Tsunoda, K., et al. 2003. Induction of pemphigus phenotype by a mouse monoclonal antibody against the amino-terminal adhesive interface of desmoglein 3. J. Immunol. 170:2170-2178.

22. Amagai, M., et al. 2000. Use of autoantigenknockout mice in developing an active autoimmune disease model for pemphigus. J. Clin. Invest. 105:625-631.

23. Stein, L.D., and Sigal, N.H. 1983. Limiting dilution analysis of Epstein Barr virus-induced immunoglobulin production. Cell. Immunol. 79:309-319.

24. Roder, J.C., Cole, S.P., and Kozbor, D. 1986. The EBV-hybridoma technique. Methods Enzymol. 121:140-167.

25. Chang, T.Y., and Siegel, D.L. 1998. Genetic and immunological properties of phage-displayed human anti-Rh(D) antibodies: implications for Rh(D) epitope topology. Blood. 91:3066-3078.

26. Ishii, K., et al. 2005. In vitro keratinocyte dissociation assay for evaluation of the pathogenicity of anti-desmoglein $3 \mathrm{IgG}$ autoantibodies in pemphigus vulgaris. J. Invest. Dermatol. In press.

27. Kljuic, A., et al. 2003. Desmoglein 4 in hair follicle differentiation and epidermal adhesion: evidence from inherited hypotrichosis and acquired pemphigus vulgaris. Cell. 113:249-260.

28. Kowalczyk, A.P., et al. 1995. Pemphigus sera recognize conformationally sensitive epitopes in the amino-terminal region of desmoglein-1 (Dsg1). J. Invest. Dermatol. 105:147-152.

29. Amagai, M., et al. 1995. Conformational epitopes of pemphigus antigens (Dsg1 and Dsg3) are calcium dependent and glycosylation independent. J. Invest. Dermatol. 105:243-247.

30. Collins, A.M., Sewell, W.A., and Edwards, M.R. 2003. Immunoglobulin gene rearrangement, repertoire diversity, and the allergic response. Pharmacol. Ther. 100:157-170.

31. Tindall, K.R., and Kunkel, T.A. 1988. Fidelity of 
DNA synthesis by the Thermus aquaticus DNA polymerase. Biochemistry. 27:6008-6013.

32. Dörner, T., et al. 1998. Delineation of selective influences shaping the mutated expressed human Ig heavy chain repertoire. J. Immunol. 160:2831-2841.

33. Futei, Y., et al. 2000. Use of domain-swapped molecules for conformational epitope mapping of desmoglein 3 in pemphigus vulgaris. J. Invest. Dermatol. 115:829-834.

34. Cook, G.P., and Tomlinson, I.M. 1995. The human immunoglobulin $\mathrm{VH}$ repertoire. Immunol. Today. 16:237-242.

35. Roark, J.H., Bussel, J.B., Cines, D.B., and Siegel, D.L. 2002. Genetic analysis of autoantibodies in idiopathic thrombocytopenic purpura reveals evidence of clonal expansion and somatic mutation. Blood. 100:1388-1398.

36. Roben, P., et al. 1996. Repertoire cloning of lupus antiDNA autoantibodies. J. Clin. Invest. 98:2827-2837.

37. Newkirk, M.M., et al. 1993. Restricted immunoglobulin variable region gene usage by hybridoma rheumatoid factors from patients with systemic lupus erythematosus and rheumatoid arthritis. Mol. Immunol. 30:255-263.

38. Dörner, T., Hansen, A., Jacobi, A., and Lipsky, P.E. 2002. Immunoglobulin repertoire analysis provides new insights into the immunopathogenesis of Sjögren's syndrome. Autoimmun. Rev. 1:119-124.

39. Adderson, E.E., Shackelford, P.G., Quinn, A., and
Carroll, W.L. 1991. Restricted Ig H chain V gene usage in the human antibody response to Haemophilus influenzae type b capsular polysaccharide. J. Immunol. 147:1667-1674.

40. Suzuki, I., Pfister, L., Glas, A., Nottenburg, C., and Milner, E.C.B. 1995. Representation of rearranged $\mathrm{VH}$ gene segments in the human adult antibody repertoire. J. Immunol. 154:3902-3911.

41. Kraj, P., et al. 1997. The human heavy chain Ig V region gene repertoire is biased at all stages of $\mathrm{B}$ cell ontogeny, including early pre-B cells. J. Immunol. 158:5824-5832.

42. Roben, P., et al. 1996. Repertoire cloning of lupus antiDNA autoantibodies. J. Clin. Invest. 98:2827-2837.

43. Goodyear, C.S., and Silverman, G.J. 2003. Death by a B cell superantigen: in vivo VH-targeted apoptotic supraclonal B cell deletion by a Staphylococcal toxin. J. Exp. Med. 197:1125-1139.

44. Silverman, G.J., Goodyear, C.S., and Siegel, D.L. 2005. On the mechanism of action of Staphylococcal protein A immunomodulation. Transfusion. 45:274-280.

45. Villard, S., et al. 2003. Peptide decoys selected by phage display block in vitro and in vivo activity of a human anti-FVIII inhibitor. Blood. 102:949-952.

46. Alvarado-Flores, E., Avalos-Diaz, E., Diaz, L.A., and Herrera-Esparza, R. 2001. Anti-idiotype antibodies neutralize in vivo the blistering effect of Pemphigus foliaceus IgG. Scand. J. Immunol. 53:254-258.

47. Grando, S.A., Pittelkow, M.R., Shultz, L.D., Dmochowski, M., and Nguyen, V.T. 2001. Pemphigus: an unfolding story. J. Invest. Dermatol. 117:990-994.

48. Barbas, C.F.I., Burton, D.R., Scott, J.K., and Silverman, G.J. 2001. Phage display: a laboratory manual. Cold Spring Harbor Laboratory Press. Cold Spring Harbor, New York, USA. 736 pp.

49. V BASE. http://vbase.mrc-cpe.cam.ac.uk. Medical Research Council Centre for Protein Engineering, University of Cambridge, Cambridge, United Kingdom.

50. Siegel, D.L., Chang, T.Y., Russell, S.L., and Bunya, V.Y. 1997. Isolation of cell surface-specific human monoclonal antibodies using phage display and magnetically-activated cell sorting: applications in immunohematology. J. Immunol. Methods. 206:73-85.

51. Amagai, M., Matsuyoshi, N., Wang, Z.H., Andl, C., and Stanley, J.R. 2000. Toxin in bullous impetigo and staphylococcal scalded-skin syndrome targets desmoglein 1. Nat. Med. 6:1275-1277.

52. Hanakawa, Y., et al. 2003. Calcium-dependent conformation of desmoglein 1 is required for its cleavage by exfoliative toxin. J. Invest. Dermatol. 121:383-389.

53. Amagai, M., Karpati, S., Prussick, R., Klaus-Kovtun, V., and Stanley, J.R. 1992. Autoantibodies against the amino-terminal cadherin-like binding domain of pemphigus vulgaris antigen are pathogenic. J. Clin. Invest. 90:919-926.

54. Hanakawa, Y., et al. 2002. Molecular mechanisms of blister formation in bullous impetigo and staphylococcal scalded skin syndrome. J. Clin. Invest. 110:53-60. doi:10.1172/JCI200215766. 